\title{
An empirical comparison of cascade and flat variants of incremental node creation algorithm
}

\author{
Sudhir Kumar Sharma • Pravin Chandra
}

Received: 31 October 2012 / Accepted: 5 September 2013/Published online: 24 September 2013

(C) CSI Publications 2013

\begin{abstract}
We propose incremental node creation algorithm (INCA). INCA emphasizes on architectural adaptation and functional adaptation in a unified frame work. INCA starts from a single hidden node and then trains node one by one incrementally. Two variants of INCA are developed, namely cascade and flat. In the cascade variant, every hidden node is added in a new hidden layer that is connected to the network inputs and all pre-existing hidden nodes. In contrast, the flat variant adds node one by one to a single hidden layer. Sixteen regression problems are used to investigate which network growing strategy provides the better generalization performance. Simulation results reveal that both architectures perform well on all the investigated regression problems of varying complexities. In general, cascade is better than flat architecture except some real world problems. The trigonometric sine activation function provides better approximation capability than log-sigmoid function except some regression problems.
\end{abstract}

Keywords Adaptive slope activation functions . Cascade neural networks - Constructive neural networks · Fourier neural networks .

Trigonometric function

\footnotetext{
S. K. Sharma $(\bowtie)$

School of Computer Science and Engineering, Ansal Institute of

Technology, Sector-55, Gurgaon, India

e-mail: sudhir_sharma99@yahoo.com

P. Chandra

University School of ICT, GGSIP University, New Delhi, India

e-mail: chandra.pravin@gmail.com
}

\section{Introduction}

The multilayer feedforward neural networks (FNNs) is popularly used class of neural network models for many applications over the past decades due to its flexibility in structure, good approximation capability, and a large number of available training algorithms $[1,2]$. The gradient-descent optimization technique based back-propagation training algorithm is extensively investigated in the literature. One of the major issues of FNNs is determining the network architecture prior to training. Recently it has been shown that the cascade network, which allows connections across layers, is more powerful than the FNNs [3]. In this paper we focus our attention on constructive neural networks $(\mathrm{CoNN})$ to determine the architecture automatically during the training process. CoNN is a collection of a group of constructive algorithms, which starts from a minimal architecture and grows the architecture during learning till near optimal architecture is achieved [4]. Many CoNN algorithms suitable for classification and regression tasks are reviewed in [5, 6], respectively. In general, a constructive algorithm grows in size by a pre-specified network growing strategy. The two well-known networkgrowing strategies are cascade-correlation network $(\mathrm{CCN})$ proposed by Fahlman and Leibere [7] and dynamic node creation (DNC) algorithm proposed by Ash [8]. The former algorithm builds cascade architecture, whereas the latter constructs single hidden layer FNN.

CCN starts the network without any hidden node. First connections' weights between input-output nodes are trained by minimizing the squared error. Algorithm adds node one by one in the current network. The hidden node is connected to all inputs and also all pre-existing hidden nodes. In the first phase, the input connection weights of the current candidate hidden node are trained to maximize 
the covariance between the candidate hidden node output and network residual error.

$S=\sum_{o}\left|\sum_{p}\left(V_{p}-\bar{V}\right)\left(e_{p, o}-\bar{e}_{o}\right)\right|$

where $p$ is the number of training patterns, $o$ is the number of output nodes, $V_{p}$ is the output of the candidate hidden node for the pattern $\mathrm{p}, e_{p, o}$ is the residual squared error at output o for pattern $\mathrm{p}$, and $\bar{V}$ and $\bar{e}_{o}$ are the corresponding averaged over all patterns.

In the second phase, current hidden node's output is connected to output nodes and then all inputs at output nodes are re-trained with the frozen input weights of all hidden nodes. In both the phases, QuickProp algorithm [9] is used for adapting the weights. The process is repeated until satisfactory solution is found or the number of hidden nodes installed reached a predefined maximum value. To prevent installation of any useless node, many candidatehidden nodes are trained independently with different random initial weights and then the best candidate node is selected and installed in the current network permanently. This feature solves weight initialization problem but makes algorithm as a computationally expensive.

The CCN has been extensively investigated in the literature and provided contradictory results on generalization capability. The generalization capability of $\mathrm{CCN}$ is sometimes cited as problematic. In [10], Sjogaard proposed a variant of standard CCN known as flat CCN, which adds new hidden nodes to a single hidden layer (cascaded connections are eliminated). The results shows that flat $\mathrm{CNN}$ gives better generalization performance in comparison to standard CCN on a single artificial problem. In [11], Phatak and Koren pointed out that standard $\mathrm{CCN}$ is more computational expensive algorithm due to increase in number of inputs of hidden nodes, as the network grows deeper. The generalization capability of $\mathrm{CCN}$ may be degraded when algorithm installs many hidden nodes, as it is likely that some short-cut connection weights may be producing intangible effect. The network depth can be drastically reduced by a simple $\mathrm{CCN}$ variant called sibling/descendant cascade-correlation network (SDCCN) as proposed by Baluja and Fahlman [12]. However, systematic comparisons show that there are no significant differences between SDCCN and standard CCN on most performance measures. In Thivierge et. al. [13], implemented optimal brain damage pruning method on both phases of standard $\mathrm{CCN}$ for classification problems. It reduced up to half of number of connections required and also improved generalization performance. In Adams and Waugh [14], reported that standard CCN produced an uneven stair-like output exhibiting poor generalization in comparison to conventional FFN on a Gaussian function approximation problem.
He pointed out that the reason of poor generalization was premature saturation of sigmoidal nodes due to constructive nature of algorithm. In Hwang [15], argued that such saturation effects are not unique to $\mathrm{CNN}$, as they are potentially present in any neural network using sigmoidal node, including conventional FNNs. As a possible solution to this problem, he proposed non-linear activation functions with trainable parameters.

Fahlman suggested the idea of cascade- 2 algorithm to others [16, 17]. Cascade-2 algorithm differs from standard $\mathrm{CCN}$ by training hidden node to directly minimize the residual error rather than to maximize its covariance with residual error. Several authors have demonstrated that $\mathrm{CCN}$ is effective for classification tasks but not very successful on regression tasks [15, 16-17]. In Prechelt [17], investigated problems and improvements in standard CCN. He developed six variants of $\mathrm{CCN}$, including cascade- 2 algorithm (cascade) and flat variant of cascade- 2 algorithm (cand). He empirically compared these variants and found that there was no significant difference between cascade and cand. In the case where there were differences in generalization, cand was superior to cascade on some problems and inferior on some others and the former case occurred more often. Standard CCN was usually inferior to cascade for regression problems and for classification problems it was often superior due to covariance criterion. In Dandurand et al. [18], compared the standard CCN and flat CCN using a student-teacher network approximation task. Experiments showed that there was no difference between the generalization capability of both flat and standard CCN but flat CCN learned more efficiently because there were fewer connections to train. $\mathrm{CCN}$ has been widely investigated and inspired the many new proposals in the literature [19-23]. The reviews of constructive algorithms that belong to CCN family are given in $[3,24]$.

There is no conclusive decision about which one is better in generalization capability, standard $\mathrm{CCN}$ or flat $\mathrm{CCN}$. The standard CNN has the following limitations: (1) $\mathrm{CNN}$ has two objective functions, one for training the input connections of candidate hidden node and other for training the output weights simultaneously. Therefore two different optimization procedures are required; (2) Covariance criterion mandates batch training and therefore stochastic optimization method cannot be used in the first phase; (3) All output weights are trained repeatedly and independently from the input training in the second phase.

An attempt to address these limitations, we propose an incremental node creation algorithm (INCA) in this paper. The algorithm emphasizes architectural adaptation and functional adaptation during training. INCA has one objective function to train input and output weights simultaneously. The two variants of INCA are developed namely cascade and flat. The difference between these 
variants is the presence/absence of connections between hidden nodes. The both variants generalize well on all investigated regression problems. These variants are used to study which network growing strategy is better in the generalization performance.

The activation functions with trainable parameters are most suitable for providing the better prediction accuracy and fast convergence [25-29]. In past, many researchers have used adaptive slope sigmoidal function in FNNs and reported faster learning and better generalization performance [30, 31]. In this paper, we exploit two activation functions with slope parameter. The log-sigmoid function (logsig) and trigonometric function (sine) are given in Table 1. The slope parameter is considered as trainable parameter and is adapted by the gradient descent method. The non-monotonic periodic trigonometric functions like sine and cosine have been used extensively in the Fourier neural networks [32]. They satisfy universal approximation theorem [33].

The rest of this paper is organized as follows: Sect. 2 describes our proposed INCA. The experimental design to compare flat and cascade architectures is given in Sect. 3. The results are presented in Sect. 4. A discussion is presented in Sect. 5. In Sect. 6, the conclusions are presented.

\section{Incremental node creation algorithm}

We propose INCA for regression problems. The single output node has a linear activation function while each hidden layers' nodes has a non-linear activation function listed in Table 1. INCA differs from the $\mathrm{CCN}$ in five aspects: (1) the INCA initializes the network with one hidden node in contrast to a direct connection between inputs and output; (2) the INCA uses only one objective function to train input and output connections simultaneously of each new hidden node. One practical advantage is that there is no need to switch between two different optimizations; (3) the INCA freezes both input and output connection weights of the trained hidden node permanently; (4) the INCA uses gradient descent method in sequential mode for training the hidden node one by one, thus we are not restricted to use batch mode training and (5) The INCA adapts the slope parameter of activation function for each pattern to improve the learning capability

Table 1 Activation functions with corresponding derivative

\begin{tabular}{lll}
\hline Label & Activation function & Derivative \\
\hline Logsig & $g(x, b)=\frac{1}{1+\exp (-b x)}$ & $\frac{\partial g}{\partial x}=b g(1-g) ; \frac{\partial g}{\partial b}=x g(1-g)$ \\
Sine & $g(x, b)=\sin (b x)$ & $\frac{\partial g}{\partial x}=b \cos (b x), \frac{\partial g}{\partial b}=x \cos (b x)$ \\
\hline
\end{tabular}

of hidden node. We develop two variants of INCA, namely cascade and flat networks to investigate the generalization capability.

\subsection{Cascade variant of INCA}

The cascade network is developed in this section. The algorithm starts from minimal architecture and trains hidden node one by one incrementally during learning. The hidden node is added in the current network that is connected to network inputs, output node and also all preexisting trained hidden nodes. Once the training of the current hidden node is completed then its input and output connection weights are frozen permanently. We assume that there is $n-1$ hidden nodes at any given point during the constructive training process. The one new $n$th hidden node is added to the current network as depicted in Fig. 1.

To reduce the residual error of the network, we choose to train input and output connection weights of this hidden node simultaneously using squared error criterion. The bias connection of output node is trained every time after adding any new hidden node in the existing network.

Let $I W_{n, i}$ represents the weight between the $n$th hidden node and $i$ th input, while $O W_{k}$ represents weight between the $k$ th hidden node and single output node. The connection weight $H I W_{n, j}$ represents weight between the $n$th hidden node and previously trained $j$ th hidden node. The weights $I W_{n, 0}$ and $O W_{0}$ act as the biases for the $n$th hidden node and output node, respectively. The biases of the hidden nodes and output node are represented using the 0th auxiliary input, $x_{0}$ and 0 th auxiliary hidden node, $O_{0}$, respectively. The values of $x_{0}$ and $O_{0}$ are set to unity.

Let the function $f$ to be approximated is given by a finite set of training pairs $\left(x^{p}, y^{p}\right) ; p=1,2, \ldots, P$; where $P$ is the number of training patterns. The index $p$ is always assumed to be present implicitly. The $n$th approximation $f_{n}$ of function $f$ will be generated incrementally for $n \geq 1$, where $n$, denotes the $n$th iteration of the algorithm.

If $x_{i}$ is the $i$ th component of the input, then net input for the $n$th hidden node is:

$n e t_{n}=\sum_{i=0}^{I N} I W_{n, i} x_{i}+\sum_{j=1}^{n-1} H I W_{n, i} O_{j}$

The output for the $n$th hidden node is:

$O_{n}=\left\{\begin{array}{l}g\left(\text { net }_{n}, b_{n}\right) n \geq 1 \\ 1 n=0\end{array}\right.$

where $g(x, b)$ is a non-linear activation function with slope parameter given in Table 1 .

A cascade network with $n$ hidden nodes, the output node implements the following function: 
Fig. 1 The Cascade architecture after adding $n$th hidden node. The connections denoted by dotted lines are only trained in the $n$th iteration

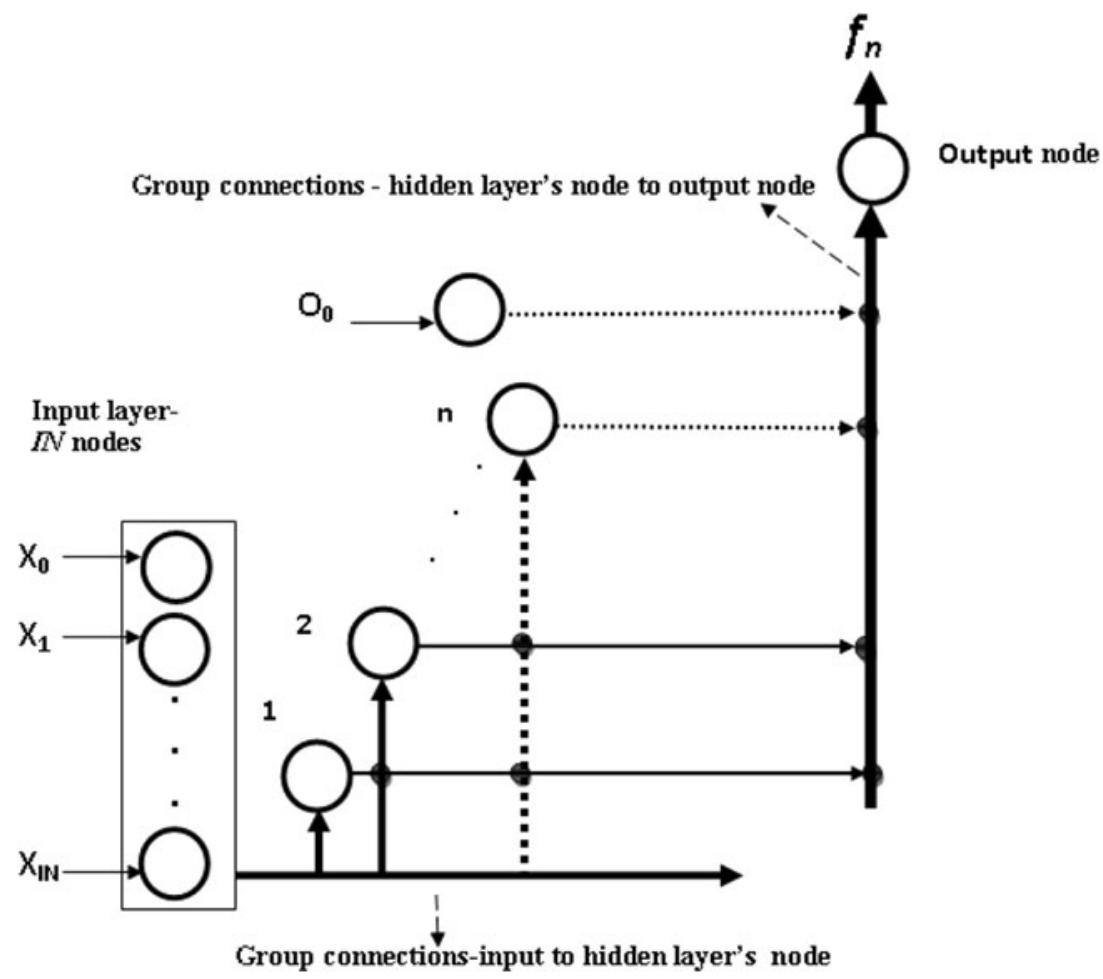

$f_{n}=\sum_{k=0}^{n} O W_{k} O_{k}=f_{n-1}(x)+F_{n}(x)$

where $f_{n-1}(x)$ is the function implemented by the cascade network that had $(n-1)$ hidden nodes and where

$F_{n}(x)=O W_{n} O_{n}+O W_{0}$

We now define the objective function for training the current $n$th hidden node by (5) that is the squared error function on a per pattern basis.

$$
\begin{aligned}
S & =\frac{1}{2}\left[f-f_{n}(x)\right]^{2}=\frac{1}{2}\left[f-f_{n-1}(x)-F_{n}(x)\right]^{2} \\
& =\frac{1}{2}\left[e_{n-1}-F_{n}(x)\right]^{2}
\end{aligned}
$$

where $e_{n-1}$ is the residual error prior to adding current hidden nodes i.e. it is the desired output for the current $n$th hidden node. The cascade network is trained by using gradient-descent method applied to the minimization of the objective function defined in (5) on a per pattern basis.

The weight increment rule can be written as follows:

$$
\Delta W(p)=-\eta_{w} \frac{\partial S}{\partial W}(p)
$$

where $W(p)$ is the weight/bias for $p$ th pattern, and $\eta_{w} \in$ $(0,1)$ is a learning constant for weight/bias. The slope parameter of activation function can be incremented as follows:

$\Delta b(p)=-\eta_{b} \frac{\partial S}{\partial b}(p)$ where $b(p)$ is the slope parameter for $p$ th pattern, and $\eta_{b} \in$ $(0,1)$ is a learning constant for slope parameter.

Let $e=\left[e_{n-1}-F_{n}(x)\right]$ be the residual error, then the increment rule for weight/bias and slope parameter are defined by (8) and (9) respectively:

$\Delta W(p)=\eta_{w} e \frac{\partial F_{n}}{\partial W}(p)$

$\Delta b(p)=\eta_{b} e \frac{\partial F_{n}}{\partial b}(p)$

Using chain rule, we can easily derive the following results:

$\frac{\partial F_{n}}{\partial O W_{k}}=O_{k} ; k=0, n$

$\frac{\partial F_{n}}{\partial I W_{n, i}}=O W_{n} \frac{\partial O_{n}}{\partial n e t_{n}} x_{i} ; i=0,1, \ldots, I N$

$\frac{\partial F_{n}}{\partial H I W_{n, i}}=O W_{n} \frac{\partial O_{n}}{\partial n e t_{n}} O_{j} ; j=0,1, \ldots, n-1$

$D O_{n}=\frac{\partial O_{n}}{\partial \text { et }_{n}}=\left\{\begin{array}{l}\partial g\left(\text { net }_{n}, b_{n}\right) / \partial \text { net }_{n} ; n \geq 1 \\ 0 ; n=0\end{array}\right.$

$D b_{n}=\frac{\partial O_{n}}{\partial b_{n}}=\left\{\begin{array}{l}\partial g\left(\text { net }_{n}, b_{n}\right) / b_{n} ; n \geq 1 \\ 0 ; n=0\end{array}\right.$

We may write the weight update rules by (15)-(18); for $n=1,2, \ldots, H N$; where $H N$ is the maximum number of 
hidden nodes added in the cascade architecture and $p=1,2, \ldots, P$.

$$
\begin{aligned}
& \Delta O W_{k}(p)=\eta_{w} \text { e }\left.O_{k}\right|_{p} ; k=0, n \\
& \Delta I W_{n, i}(p)=\eta_{w} \text { e }\left.O W_{n} D O_{n} x_{i}\right|_{p} ; i=0,1, \ldots, I N \\
& \Delta H I W_{n, j}(p)=\eta_{w} \text { e }\left.O W_{n} D O_{n} O_{j}\right|_{p} ; j=1, \ldots, n-1
\end{aligned}
$$

$\Delta b_{n}(p)=\eta_{b}$ e $\left.O W_{n} D b_{n}\right|_{p}$

\subsection{Flat variant of INCA}

The flat variant of INCA adds node one by one in a single hidden layer incrementally. Flat network do not contain connections between hidden nodes. In the $n$th iteration, $n$th node is added in the current network as depicted in Fig. 2. The connections denoted by dotted lines are only trained in the $n$th iteration. The algorithm for flat network can be easily derived from the cascade network as discussed in Sect. 2.1 by eliminating the connections between the hidden nodes.

In this paper, we compare the generalization performance of cascade and flat networks to investigate the role of network growing strategies.

\section{Experimental design}

A methodology for comparative performance between cascade and flat networks is based on the prediction accuracy (generalization capability) of trained network on independent testing set. For fair evaluation of the generalization capability of two variants of INCA, the benchmark regression problems from the literature are considered. We have chosen 16 different regression problems of varying complexities

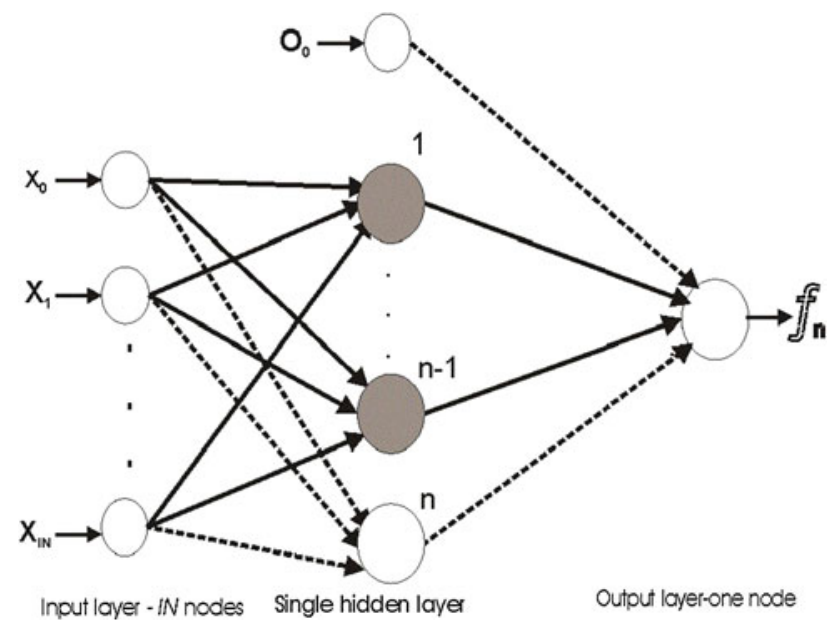

Fig. 2 The Flat architecture after adding $n$th hidden node such as simple, radial function, harmonic function, complicated interaction function, time-series prediction and some real world problems [19, 21, 34-38]. We have considered 5 real world problems (1)-(5), and 11 artificial function approximation problems (6)-(10).

(1) Boston Housing Regression Problem (Housing):

We consider Boston Housing regression problem from UCI database [37]. The problem is to predict the housing values in suburbs of Boston using thirteen attributes of house.

(2) Auto MPG (Mpg):

The second problem is Auto MPG regression problem taken from UCI database [37]. The problem is to prediction of fuel consumption in miles per gallon for different models of cars.

(3) Chaotic Henon Map (Henon):

The Henon map is a time-series prediction problem. The chaotic time series data is defined as

$x_{t+1}=1+\alpha x_{t}^{2}+\beta x_{t-1}$

where $x_{t}$ is the value at time $t$. The values of coefficient are $\alpha=-1.4$ and $\beta=0.3$. The initial values for the time series are $x_{0}=1.0$, and $x_{1}=0.4$ [20]. The time series of 300 data points is used in the experiments.

(4) Laser time series (Laser):

The laser series problem contains 10,000 data points of intensity fluctuations of laser recorded from a far-infrared laser in a physics laboratory [38]. We used first 3,010 data points. The first 10 data points used to predict the 11th data point represent one instance. The first 1000 instances are used for training, next 1000 are used for validation, and remaining 1000 is used for testing data sets.

(5) Mackey-Glass chaotic time-series (Mackey):

The Mackey-Glass is a chaotic time-series prediction problem in which data points are generated by the following differential equation

$\frac{d x(t)}{d t}=\frac{\alpha x(t-T)}{1+x(t-T)^{10}}-\beta x(t)$

where the parameters $\alpha=0.2, \beta=0.1$, and $T=17$. Fourth order Runge-Kutta method is used to generate 2000 data points. The time step used in this method is 0.1 and the initial conditions are $x(0)=1.2, x(t)=0$, for $t<0$. We use the four values $x(t-18), x(t-12), x(t-6), x(t)$ to predict $x(t+6)$. Thus, the number of inputs in network is four and the number of outputs is one. The data points are used in the range of $[101,1624]$.

(6) 1-D Problems: We consider the following two onedimensional regression problems.

- Sinc function (SincF)

$y=\sin x / x ; x \in(0,2 \pi)$

- Simple function (SF) 


$$
\begin{aligned}
y= & 0.2\left\{1+0.1(x-7)^{2}\right\} \cos (2 x)+0.5 e^{-2 x} \\
& \times \sin (2 x-0.1 \pi) ; x \in(0,1)
\end{aligned}
$$

(7) 2-D Problems: We consider the following five twodimensional regression problems defined in domain $[0,1]$.

- Simple interaction function (SIF)

$y=10.391\left[\left(x_{1}-0.4\right)\left(x_{2}-0.6\right)+0.36\right]$;

Radial function (RF)

$$
\begin{aligned}
y= & 24.234\left[\left\{\left(x_{1}-0.5\right)^{2}+\left(x_{2}-0.5\right)^{2}\right\}\right. \\
& \left.\left\{0.75-\left\{\left(x_{1}-0.5\right)^{2}+\left(x_{2}-0.5\right)^{2}\right\}\right\}\right] ;
\end{aligned}
$$

- Harmonic function (HF)

$$
\begin{aligned}
y= & 42.659\left[0.1+\left(x_{1}-0.5\right)\left\{0.05-10\left(x_{1}-0.5\right)^{2}\right.\right. \\
& \left.\left.\left(x_{2}-0.5\right)^{2}+\left(x_{1}-0.5\right)^{4}+5\left(x_{2}-0.5\right)^{4}\right\}\right] ;
\end{aligned}
$$

- Additive function (AF)

$$
\begin{aligned}
y= & 1.3356\left[1.5\left(1-x_{1}\right)+e^{2 x_{1}-1} \sin \left\{3 \pi\left(x_{1}-0.6\right)^{2}\right\}\right. \\
& \left.+e^{3\left(x_{2}-0.5\right)} \sin \left\{4 \pi\left(x_{2}-0.9\right)^{2}\right\}\right] ;
\end{aligned}
$$

- Complicated interaction function (CIF)

$y=1.9\left[1.35+e^{x_{1}} \sin \left\{13\left(x_{1}-0.6\right)^{2}\right\} e^{-x_{2}} \sin \left(7 x_{2}\right)\right]$;

(8) SAF Problem: We consider the following threedimensional regression problem [34].

$$
y=\frac{1}{1+\exp \left[-e^{x_{1}}+\left(x_{2}-0.5\right)^{2}+3 \sin \left(\pi x_{3}\right)\right]} ;
$$

$\mathrm{X}$ is uniform in $[0,1]$

(9) 4-D Problems: We consider the following two fourdimensional regression problems.

- Additive function called (CAF)

$$
\begin{aligned}
y= & \exp \left\{2 x_{1} \sin \left(\pi x_{4}\right)\right\} \\
& +\sin \left(x_{2} x_{3}\right) ; X \text { is uniform in }[-0.25,0.25]
\end{aligned}
$$

- Powell's quadratic function called (PQF)

$$
\begin{aligned}
y= & \left(x_{1}+10 x_{2}\right)^{2}+5\left(x_{3}-x_{4}\right)^{2}+\left(x_{2}-2 x_{3}\right)^{4} \\
& +10\left(x_{1}-x_{4}\right) ; \mathrm{X} \text { uniform in }[-1,1]
\end{aligned}
$$

(10) 5DRF: We consider the following five-dimensional regression problem [36].

$y=\frac{x_{1} x_{2} x_{3} x_{5}\left(x_{3}-1\right)+x_{4}}{1+x_{2}^{2}+x_{3}^{2}} ; \mathrm{X}$ is uniform in $[-1,1]$

The training data for all regression problems (except 1-5 problems) are generated randomly in a given domain. The entire data is normalized in the interval $[-1,1]$ for all problems. The data is partitioned into training set (trS), validation set (vaS), and testing set (teS), in sequence. The hidden nodes are added and trained one by one up to a maximum of 15 as shown by $H N$. Each individual hidden node is trained for up to a maximum of 200 epochs as shown by $(\tau)$. These two parameters are selected empirically based on the complexity of a given problem. The summary of the data sets used and other training parameters for all regression problems are given in Table 2. Columns 2 and 3 show number of inputs and outputs respectively. Columns 4, 5 and 6 show the number of training, validation and testing data respectively. The $H N$ and $\tau$ are given in columns 7 and 8 , respectively.

The learning rate $\eta_{w}$ and $\eta_{b}$ are set to a default value, 0.1 and 0.05 respectively for all the experiments. The $t r S$ is used to update the weights of newly added hidden node during constructive training process. The $v a S$ is used to assess the generalization performance of the network after completing the training of each hidden node. The $t e S$ is used to predict the generalization performance of selected network (same configuration of the network, where mean squared error (MSE) at vaS is minimum during training). 30 independent trials are performed for each regression problem. For each trial, initial weights are randomly generated in the interval $[-0.5,0.5]$ for each hidden node. We started slope parameters of both activation functions with a value of unity and updated them so that they reached their optimal values. For better use of non-linearity, we restricted the slope parameter to be more than 0.2.

\section{Experimental results}

This section presents the results of all experiments conducted in this paper. We first evaluate and compare the performance of both cascade and flat networks with backpropagation algorithm in sequential mode (BP) [33] and extreme learning machine (ELM) [40] for Housing and Mpg problems. Recently, ELM has been evolved fast and better generalization algorithm for regression and classification problems. The following measures were used for comparison of generalization performance using logsig and sine activation functions:

1. The mean (Mean) and standard deviation (S.D.) of the MSE on teS achieved in 30 trials.

2. The mean (Mean) and standard deviation (S.D.) of number of hidden nodes installed by algorithm in 30 trials.

Table 3 summarizes all results obtained from the experiments conducted over four algorithms on two real world problems. 
Table 2 Summary of the data sets and other training parameters

\begin{tabular}{lclccccc}
\hline Problems & \# Inputs & \# Outputs & \# Training data & \# Validation data & \# Testing data & HN & $\tau$ \\
\hline Housing & 13 & 1 & 401 & 80 & 25 & 15 & 200 \\
Mpg & 7 & 1 & 300 & 46 & 46 & 15 & 200 \\
Henon & 2 & 1 & 100 & 100 & 100 & 15 & 150 \\
Laser & 10 & 1 & 1000 & 1000 & 1000 & 15 & 150 \\
Mackey & 4 & 1 & 500 & 500 & 500 & 10 & 200 \\
1-D problems & 1 & 1 & 100 & 100 & 1000 & 5 & 25 \\
2-D problems & 2 & 1 & 225 & 225 & 1000 & 15 & 200 \\
SAF & 3 & 1 & 300 & 300 & 1000 & 15 & 200 \\
4-D problems & 4 & 1 & 400 & 400 & 1000 & 15 & 200 \\
5DRF & 5 & 1 & 500 & 500 & 1000 & 15 & 200 \\
\hline
\end{tabular}

Table 3 Summary results of four algorithms using logsig and sine activation function

\begin{tabular}{|c|c|c|c|c|c|c|c|c|c|}
\hline \multirow[t]{3}{*}{ Problem } & \multirow[t]{3}{*}{ Algorithm } & \multicolumn{4}{|c|}{ Logsig activation function } & \multicolumn{4}{|c|}{ Sine activation function } \\
\hline & & \multicolumn{2}{|c|}{$\operatorname{MSE}\left(10^{-2}\right)$ at teS } & \multicolumn{2}{|c|}{ Hidden nodes } & \multicolumn{2}{|c|}{$\operatorname{MSE}\left(10^{-2}\right)$ at teS } & \multicolumn{2}{|c|}{ Hidden nodes } \\
\hline & & Mean & S.D. & Mean & S.D. & Mean & S.D. & Mean & S.D. \\
\hline \multirow[t]{4}{*}{ Housing } & Cascade & 2.2871 & 0.2193 & 6.333 & 3.567 & 2.0093 & 0.4926 & 7.833 & 4.624 \\
\hline & Flat & 2.2183 & 0.2302 & 7.967 & 3.672 & 1.8602 & 0.3406 & 6.067 & 4.835 \\
\hline & BP & 3.4464 & 1.4425 & 15.00 & 0.000 & 4.3353 & 2.1737 & 15.00 & 0.00 \\
\hline & ELM & 2.5575 & 0.5143 & 55.00 & 0.000 & 2.7221 & 0.5759 & 55.00 & 0.00 \\
\hline \multirow[t]{4}{*}{ Mpg } & Cascade & 1.9061 & 0.0592 & 4.800 & 4.693 & 1.7930 & 0.0633 & 5.667 & 4.294 \\
\hline & Flat & 1.8753 & 0.0414 & 4.9667 & 4.552 & 1.7782 & 0.0426 & 5.967 & 4.198 \\
\hline & $\mathrm{BP}$ & 1.8977 & 0.5059 & 15.00 & 0.000 & 1.8335 & 0.3045 & 15.00 & 0.00 \\
\hline & ELM & 2.1024 & 0.3237 & 55.00 & 0.000 & 2.1891 & 0.2466 & 55.00 & 0.000 \\
\hline
\end{tabular}

It is obvious from the Table 3 , the cascade and the flat networks perform better than BP and ELM for Housing problem. There is no significant difference between all four algorithms for Mpg problem and cascade and flat networks for both the problems. The flat network seems better than cascade for both real world problems. The major focus of this paper is investigating the prediction accuracy of cascade and flat variants. Table 4 presents the summary of simulation results for both variants of INCA using logsig and sine activation functions on 14 problems.

For further analysis of the results, we have used paired $t$-test statistical inference method to test which network growing strategy: cascade/flat is significantly better in generalization performance. The alternate hypothesis for $t$-test is: "is cascade better than flat architecture" on the basis of mean MSE on teS. The results of $t$-test for both variants are presented in Table 5. The $p$-value (probability) obtained with right tailed $t$-test and $t$-value (test statistic) is given. If $p$-value is less than 0.05 , then alternative hypothesis is accepted at $95 \%$ confidence level. In the table, the test cases for which flat architecture is better than cascade architecture are highlighted in bold print and the test cases for which there is no significance difference between cascade and flat architecture are underlined.

To establish the generalization capability of best architecture, $t$-test results given in table 5 are further summarized as in Table 6.

1. The number of regression problems in which, the cascade architecture is significantly better than the flat architecture is given in second column.

2. The number of regression problems in which, there is no significance difference between the cascade and the flat architectures is given in the third column.

3. The number of regression problems in which, the flat architecture is significantly better than the cascade architecture is given in the fourth column.

On the basis of simulation results, we can conclude as follows:

1. Both the cascade and the flat architectures performed well on all investigated regression problems of varying complexities.

2. In general, cascade architecture has better generalization performance as compared to flat architecture. 
Table 4 Summary results of the cascade and flat architectures using logsig and sine activation function

\begin{tabular}{|c|c|c|c|c|c|c|c|c|c|}
\hline \multirow[t]{3}{*}{ Problem } & \multirow[t]{3}{*}{ Algorithm } & \multicolumn{4}{|c|}{ Logsig activation function } & \multicolumn{4}{|c|}{ Sine activation function } \\
\hline & & \multicolumn{2}{|c|}{ MSE $\left(10^{-2}\right)$ at teS } & \multicolumn{2}{|c|}{ Hidden nodes } & \multicolumn{2}{|c|}{$\operatorname{MSE}\left(10^{-2}\right)$ at teS } & \multicolumn{2}{|c|}{ Hidden nodes } \\
\hline & & Mean & S.D. & Mean & S.D. & Mean & S.D. & Mean & S.D. \\
\hline \multirow[t]{2}{*}{ Henon } & Cascade & 0.9822 & 0.4071 & 11.767 & 3.683 & 0.1116 & 0.072 & 13.300 & 2.480 \\
\hline & Flat & 1.4797 & 0.1072 & 9.200 & 3.556 & 0.2144 & 0.0152 & 2.333 & 1.493 \\
\hline \multirow[t]{2}{*}{ Laser } & Cascade & 1.0762 & 0.2344 & 11.467 & 3.181 & 0.5725 & 0.2207 & 13.467 & 1.833 \\
\hline & Flat & 1.4035 & 0.4986 & 12.167 & 3.097 & 1.1436 & 0.2988 & 11.200 & 3.067 \\
\hline \multirow[t]{2}{*}{ Mackey } & Cascade & 0.2264 & 0.0352 & 3.700 & 2.973 & 0.1363 & 0.1200 & 6.400 & 3.201 \\
\hline & Flat & 0.2212 & 0.0372 & 3.133 & 2.596 & 1.4034 & 3.0298 & 5.100 & 3.356 \\
\hline \multirow[t]{2}{*}{$\operatorname{SinF}$} & Cascade & 0.6298 & 0.0323 & 4.033 & 1.159 & 0.1163 & 0.0031 & 2.433 & 1.654 \\
\hline & Flat & 0.7236 & 0.0084 & 3.400 & 1.221 & 0.1175 & 0.0015 & 2.267 & 1.337 \\
\hline \multirow[t]{2}{*}{ SF } & Cascade & 0.1325 & 0.0130 & 3.233 & 1.407 & 0.0107 & 0.0023 & 2.467 & 1.432 \\
\hline & Flat & 0.1031 & 0.0055 & 2.600 & 1.589 & 0.0111 & 0.0018 & 3.233 & 1.073 \\
\hline \multirow[t]{2}{*}{ SIF } & Cascade & 0.2646 & 0.0448 & 12.200 & 3.078 & 0.0509 & 0.0347 & 14.233 & 1.278 \\
\hline & Flat & 0.5036 & 0.0624 & 9.500 & 3.646 & 0.1609 & 0.0812 & 12.867 & 2.898 \\
\hline \multirow[t]{2}{*}{ RF } & Cascade & 3.1014 & 0.9132 & 11.567 & 3.159 & 0.3988 & 0.2949 & 13.733 & 1.893 \\
\hline & Flat & 4.2586 & 0.3192 & 13.433 & 2.431 & 2.6734 & 0.599 & 8.933 & 5.009 \\
\hline \multirow[t]{2}{*}{$\mathrm{HF}$} & Cascade & 5.3579 & 0.3955 & 5.633 & 3.625 & 6.4429 & 2.8074 & 9.067 & 4.792 \\
\hline & Flat & 5.4590 & 0.4672 & 10.800 & 4.139 & 5.9923 & 0.865 & 8.067 & 4.168 \\
\hline \multirow[t]{2}{*}{$\mathrm{AF}$} & Cascade & 2.0867 & 0.2926 & 10.100 & 4.221 & 0.7406 & 0.3385 & 14.567 & 0.898 \\
\hline & Flat & 3.2852 & 0.6748 & 9.500 & 3.770 & 2.7326 & 0.2601 & 8.933 & 2.888 \\
\hline \multirow[t]{2}{*}{ CIF } & Cascade & 3.8367 & 0.8098 & 9.733 & 4.059 & 2.004 & 1.1334 & 13.700 & 2.395 \\
\hline & Flat & 5.3406 & 0.5290 & 10.900 & 2.759 & 3.7254 & 0.7933 & 11.733 & 2.864 \\
\hline \multirow[t]{2}{*}{ SAF } & Cascade & 1.1000 & 0.1276 & 9.967 & 3.378 & 0.1793 & 0.0484 & 14.567 & 0.858 \\
\hline & Flat & 1.0673 & 0.1243 & 11.533 & 2.675 & 0.4659 & 0.0432 & 12.433 & 2.725 \\
\hline \multirow[t]{2}{*}{ CAF } & Cascade & 0.5897 & 0.0804 & 14.267 & 1.285 & 0.1651 & 0.4922 & 14.200 & 2.413 \\
\hline & Flat & 0.9495 & 0.1021 & 11.300 & 3.640 & 0.2119 & 0.0604 & 12.700 & 1.968 \\
\hline \multirow[t]{2}{*}{ PQF } & Cascade & 0.6792 & 0.2008 & 13.767 & 1.870 & 0.1161 & 0.0135 & 13.600 & 1.632 \\
\hline & Flat & 1.3342 & 0.3707 & 13.000 & 2.117 & 0.2767 & 0.0285 & 13.300 & 1.803 \\
\hline \multirow[t]{2}{*}{$5 \mathrm{DRF}$} & Cascade & 1.6306 & 0.0094 & 8.467 & 4.912 & 1.4968 & 0.0878 & 5.200 & 5.397 \\
\hline & Flat & 1.6400 & 0.0050 & 9.267 & 4.675 & 1.3486 & 0.2255 & 9.167 & 4.534 \\
\hline
\end{tabular}

3. There is no significant performance difference between the flat and the cascade architectures for real world problems. For time series problems cascade is better than flat architecture.

The both variants of INCA employ a greedy approach to construct feedforward network. The algorithm trains hidden node one by one incrementally. We assume that trained hidden nodes in the network are useful in finding the unknown mapping from the training data. Therefore, we froze the input and output weights of existing hidden nodes, and allowed the adaptation in input and output weights of new hidden node. To investigate the reason of better performance of cascade architecture, the sum of mean of hidden nodes for all investigated regression problems is presented in Table 7.

The generalization performance of the cascade architecture is better than the flat architecture for sine activation function because cascade network installs more hidden nodes as compared to flat architecture. For logsig activation function, the installed number of hidden nodes is approximately equal for both the architectures. This indicates that the cascade architecture is better choice due to the participation of short-cut connections in the learning. The regression problems where training time is a major consideration, the flat architecture may be preferred due to the absence of short-cut connections between hidden nodes. Therefore the flat architecture is more computationally efficient.

To establish the suitability of activation functions for cascade and the flat architectures, we have used rank method on Mean MSE. The activation function producing lowest Mean MSE is assigned a rank 1, and the other is assigned a rank 0 . The rank-sum of all investigated regression problems for both architectures is given in first and second rows of Table 8 . 
Table 5 t-test comparison results of cascade and flat variant of INCA

\begin{tabular}{|c|c|c|c|c|}
\hline \multirow[t]{2}{*}{ Problem } & \multicolumn{2}{|l|}{ Logsig } & \multicolumn{2}{|l|}{ Sine } \\
\hline & $p$-Value & $t$-Value & $p$-Value & $t$-Value \\
\hline Housing & $\underline{0.803}$ & -1.21 & $\underline{0.898}$ & -1.299 \\
\hline Mpg & $\underline{0.979}$ & -2.15 & $\underline{0.845}$ & -1.034 \\
\hline Henon & 0.000 & 6.15 & 0.000 & 8.023 \\
\hline Laser & 0.003 & 2.97 & 0.000 & 8.970 \\
\hline Mackey & $\underline{0.598}$ & -0.533 & 0.014 & 2.309 \\
\hline SincF & 000 & 17.14 & 0.033 & 1.918 \\
\hline SF & 1 & -10.61 & $\underline{0.251}$ & $\underline{0.678}$ \\
\hline SIF & 0.000 & 16.74 & 0.000 & 6.853 \\
\hline $\mathrm{RF}$ & 0.000 & 6.08 & $\underline{0.200}$ & $\underline{0.833}$ \\
\hline $\mathrm{HF}$ & $\underline{0.159}$ & $\underline{1.02}$ & $\underline{0.798}$ & -0.847 \\
\hline $\mathrm{AF}$ & 0.000 & 7.79 & 0.000 & 25.00 \\
\hline CIF & 0.000 & 7.95 & 0.000 & 7.869 \\
\hline SAF & $\underline{0.858}$ & -1.09 & 0.000 & 27.12 \\
\hline CAF & 0.000 & 16.06 & $\underline{0.300}$ & $\underline{0.510}$ \\
\hline $\mathrm{PQF}$ & 0.000 & 8.92 & 0.000 & 28.40 \\
\hline $5 \mathrm{DRF}$ & 0.000 & 5.09 & 0.987 & -3.067 \\
\hline
\end{tabular}

Table 6 Summery of $t$-test results

\begin{tabular}{llll}
\hline $\begin{array}{l}\text { Activation } \\
\text { function }\end{array}$ & $\begin{array}{l}\text { Cascade } \\
\text { (better) }\end{array}$ & $\begin{array}{l}\text { No decision } \\
\text { (equivocal) }\end{array}$ & $\begin{array}{l}\text { Flat } \\
\text { (better) }\end{array}$ \\
\hline Logsig & 10 & 5 & 1 \\
Sine & 9 & 6 & 1 \\
\hline
\end{tabular}

Table 7 Sum of mean of hidden nodes

\begin{tabular}{lll}
\hline Activation function & \multicolumn{2}{l}{ Total of mean of hidden nodes } \\
\cline { 2 - 3 } & Cascade & Flat \\
\hline Logsig & 141.034 & 142.507 \\
Sine & 164.43 & 134.30 \\
\hline
\end{tabular}

Table 8 Summery results for comparison of activation functions

\begin{tabular}{lll}
\hline Architecture & \multicolumn{2}{l}{ Rank-sum on mean MSE } \\
\cline { 2 - 3 } & Logsig & Sine \\
\hline Cascade & 1 & 15 \\
Flat & 2 & 14 \\
\hline
\end{tabular}

In general, sine function provides better approximation capability for both cascade and the flat architectures except $H F$ problem. The logsig activation function provides better results only for $H F$, and Mackey problems.

\section{Discussions}

Previous research provides contradictory conclusion on generalization capability of standard $\mathrm{CCN}$ for regression problems. Whereas our simulation results shows that cascade architecture generalize well on regression problems of varied complexities and time-series prediction problems. Previous research did not find out the conclusive decision about generalization capabilities of standard $\mathrm{CCN}$ and flat CCN. Littman and Ritter suggested that standard CCN generalized better than the flat $\mathrm{CCN}$ whereas Sjogaard suggested that the flat $\mathrm{CCN}$ was a better choice than the standard CCN $[39,10]$. Prechelt empirically found that the flat variant was superior to the cascade variant for some problems [17]. Dandurand et al. [18] concluded that there was no difference between the standard $\mathrm{CCN}$ and the flat $\mathrm{CNN}$ in terms of generalization performance. Our simulation results show that both cascade and flat variants of INCA generalize well on regression problems of varied complexities and time-series prediction problems. According to our results, the cascade architecture is superior in generalization performance than the flat architecture for problems of varied complexities and time-series prediction problems. In general, the shortcut-connections between hidden nodes are contributing to provide better approximation capability. There is no significant difference between cascade and flat architectures for Housing and Mpg problems, but flat architecture seems better than cascade architecture for both real world problems.

Adams and Waugh reported that standard CCN exhibits poor generalization for sigmoidal hidden nodes [14]. Our simulation results also show that using logsig activation function, the generalization performance of both architectures are inferior than using sine activation function. The logsig provides better result for Mackey and Harmonic regression function. The suitability of sine activation function on INCA needs to be further analyzed theoretically.

We suggest that further research is necessary to determine the utility of short-cut connections in standard CCN. In this paper, we investigated two extreme network growing strategies, one fully cascaded and another completely flat. Further research could explore more flexible network growing strategies such as SDCCN. In addition, pruning method could be used to remove surplus cascading connections to take advantage of computationally efficient flat architecture and using cascading connections in building powerful-cascaded feature detectors.

\section{Conclusion}

This paper presented an empirical comparison between cascade and flat variants of INCA using log-sigmoid and sine activation functions with adaptive slope parameter for sixteen regression problems. The simulation results revealed that the both flat and cascade variants performed well on all investigated regression problems of varied 
complexities. In general, cascade network was superior in generalization performance than the flat network. There was no significant difference between both architectures for real world problems. The sine activation function provided better performance for both architectures, except some regression problems.

Acknowledgments The authors gratefully acknowledge the contribution of the anonymous reviewer's comments in improving the presentation of the work.

\section{References}

1. Cybenko G (1989) Approximation by superpositions of a sigmoidal function. Math Control Signal Syst 2:303-314

2. Hornik K, Stinchcombe M, White H (1989) Multilayer feedforward networks are universal approximators. Neural Netw 2(5):359-366

3. Wilamowski BM (2009) Neural network architectures and learning algorithms. IEEE Ind Electron Mag 3(4):56-63

4. Sharma SK, Chandra P (2010) Constructive neural networks: a review. Int J Eng Technol 2(12):7847-7855

5. Nicoletti MC et al. (2009) Constructive neural network algorithm for feedforward architectures suitable for classification tasks. In: Leonardo Franco etc. (ed): Constructive neural networks (Studies in Computational Intelligence vol. 258), pp 1-23

6. Kwok TY, Yeung DY (1997) Constructive algorithms for structure learning in feedforward neural networks for regression problems. IEEE Trans Neural Netw 8(3):630-645

7. Fahlman SE, Lebiere C (1990) The cascade correlation learning architecture. In: Proc. Advances Neural Inform Process Syst 2:524-532

8. Ash T (1989) Dynamic node creation in backpropagation networks. Connec Sci 1(4):365-375

9. Fahlman SE (1988) Faster-learning variations on back-propagation: an empirical study. In: Sejnowski TJ, Hinton GE, Touretzky DS (eds) Proceedings of connectionist models summer school. Morgan Kaufmann, San Mateo

10. Sjogaard S (1991) A conceptual approach to generalization in dynamic neural networks. Ph.D. thesis, Computer Science Department, Aarhus University, Denmark

11. Phatak DS, Koren I (1994) Connectivity and performance tradeoffs in the cascade correlation learning architecture. IEEE Trans Neural Netw 5(6):930-935

12. Baluja S, Fahlman SE (1994) Reducing network depth in the cascade-correlation. Technical report CMU-CS-94-209, Pittsburgh, Carnegie Mellon University

13. Thivierge JP, Rivest F, Shultz TR (2003) A dual-phase technique for pruning constructive networks. In: proceedings of the IEEE International Joint Conference on Neural Networks, vol. 1, pp 559-564

14. Adams A, Waugh S (1995) Function evaluation and the cascadecorrelation architecture. In: The IEEE International Conference on Neural Networks, pp 942-946

15. Hwang JN, Shien S, Lay SR (1996) The Cascade - correlation learning: a projection pursuit learning perspective. IEEE Trans Neural Netw 7(2):278-289

16. Nechyba MC, Xu Y (1994) Neural network approach to control system identification with variable activation functions. IEEE International Symposium on Intelligent Control, Columbus, Ohio, USA

17. Prechelt L (1997) Investigation of the cascor family of learning algorithms. Neural Netw 10(5):885-896
18. Dandurand F, Berthiaume V, Shultz TR (2007) A systematic comparison of flat and standard cascade-correlation using a student-teacher network approximation task. Connection Science 19(13):223-244

19. Kwok TY, Yenug DY (1997) Objective functions for training new hidden units in constructive neural networks. IEEE Trans Neural Netw 8(5):1131-1148

20. Lehtokangas M (1999) Modeling with constructive backpropagation. Neural Netw 12:707-716

21. Treadgold NK, Gedeon TD (1999) Exploring constructive cascade networks. IEEE Trans Neural Netw 10(6):1335-1350

22. Ma L, Khorasani K (2003) A new strategy for adaptively constructing multilayer feedforward neural networks. Neurocomputing 51:361-385

23. Huang G, Shiji S, Cheng W (2012) Orthogonal least squares algorithm for training cascade neural networks. IEEE Trans on Circuits and Systems, 59II:2629-2637

24. Lahnajarvi JJT, Lehtokangas MI, Saarinen JPP (2002) Evaluation of constructive neural networks with cascaded architectures. Neurocomputing 48:573-607

25. Vecci L, Piazza F, Uncini A (1998) Learning and approximation capabilities of adaptive spline activation function neural networks. Neural Netw 11:259-270

26. Trentin E (2001) Networks with trainable amplitude of activation functions. Neural Netw 14:471-493

27. Zhang M, Xu S, Fulcher J (2002) Neuron-adaptive higher order neural-network models for automated financial data modeling. IEEE Trans Neural Netw 13(1):188-204

28. Chandra P, Singh Y (2003) An activation function adapting training algorithm for sigmoidal feedforward networks. Neurocomputing 6:429-437

29. Castelli I, Trentin E (2013) Combination of supervised and unsupervised learning for training the activation functions of neural networks", Pattern Recognition Letters, Elsevier, http://dx. doi.org/10.1016/j.patrec.2013.06.013

30. Hu Z, Shao H (1992) The study of neural network adaptive control systems. Control Decis 7:361-366

31. Chen CT, Chang WD (1996) A feedforward neural network with function shape autotuning. Neural Netw 9(4):627-641

32. Gallant AR, White H (1988) There exists a neural network that do not make avoidable mistakes. In: Proc. IEEE Conf. Neural Network, San Diego, vol 1, pp. 567-664

33. Gupta MM et al (2004) Static and dynamic neural networks: from fundamentals to advanced theory, vol chapter 7. Wiley, New York, pp 253-323

34. Samad T (1991) Backpropagation with expected source values. Neural Netw 4(5):615-618

35. Cherkassky V, Gehring D, Mulier F (1996) Comparison of adaptive methods for function estimation from samples. IEEE Trans Neural Netw 7(4):969-984

36. Narendra KS, Parthasarathy K (1990) Identification and control of dynamical system using neural networks. IEEE Trans Neural Netw 1:4-27

37. The UCI Machine Learning Repository WWW Site, (http://www. ics.uci.edu/ $\sim$ mlearn/MLRepository.html)

38. The Santa Fe Time Series Competition Data WWW site (http:/ www.stern.nyu.edu/ aweigend/Time_Series/SantaFe.html)

39. Littmann E, Ritter H (1993) Generalization abilities of cascade network architectures. In: SJ Hanson, J Cowan, CL Giles (eds), Advances in Neural Information Processing System 5, Morgan Kaufmann, pp 188-195

40. Huang GB, Zhu QY, Siew CK (2004) Extreme learning machine: a new learning scheme of feedforward neural networks. In: Proc. International Joint Conference on Neural Networks (IJCNN'2004), pp 985-990 